\title{
A Hyper Heuristic Algorithm to Solve the Low-Carbon Location Routing Problem
}

\author{
Chunmiao Zhang ${ }^{1,2}\left(\mathbb{D}\right.$, Yanwei Zhao ${ }^{1, *} \mathbb{(}$ and Longlong Leng ${ }^{1(1)}$ \\ 1 Key Laboratory of Special Equipment Manufacturing and Advanced Processing Technology, \\ Ministry of Education, Zhejiang University of Technology, Hangzhou 310023, China \\ 2 Mechanical and Automotive Branches, Jiaxing Vocational and Technical College, Jiaxing 314036, China \\ * Correspondence: ywz@zjut.edu.cn
}

Received: 25 May 2019; Accepted: 24 June 2019; Published: 27 June 2019

\begin{abstract}
This paper proposes a low-carbon location routing problem (LCLRP) model with simultaneous delivery and pick up, time windows, and heterogeneous fleets to reduce the logistics cost and carbon emissions and improve customer satisfaction. The correctness of the model is tested by a simple example of CPLEX (optimization software for mathematical programming). To solve this problem, a hyper-heuristic algorithm is designed based on a secondary exponential smoothing strategy and adaptive receiving mechanism. The algorithm can achieve fast convergence and is highly robust. This case study analyzes the impact of depot distribution and cost, heterogeneous fleets (HF), and customer distribution and time windows on logistics costs, carbon emissions, and customer satisfaction. The experimental results show that the proposed model can reduce logistics costs by $1.72 \%$, carbon emissions by $11.23 \%$, and vehicle travel distance by $9.69 \%$, and show that the proposed model has guiding significance for reducing logistics costs.
\end{abstract}

Keywords: regional low-carbon location-routing problem; time windows; heterogeneous fleets; hyper-heuristic

\section{Introduction}

The location-routing problem (LRP) is one of the most important combinatorial optimization problems in supply chain management and logistics system planning [1]. Its processing directly affects logistics distribution costs and efficiency, customer satisfaction, and environmental issues. Low-carbon LRP (LCLRP) [2] is the problem of driving route planning and depot location while considering carbon emission factors. In recent years, vehicle routing problems and depot location issues have become increasingly important for the economy, society, and the environment. Therefore, we construct a mathematical model of regional LCLRP, taking into consideration time windows, simultaneous pickup and delivery with heterogeneous fleets (RLCLRPTWSPDHF). Its purpose is to analyze the factors affecting logistics costs, vehicle carbon emissions, and customer satisfaction. Simultaneously, a novel hyper-heuristic $(\mathrm{HH})$ algorithm was developed by applying the second exponential smoothing method (SESM) combined with an adaptive mechanism as hyper-level selection strategies, and a self-adaptive acceptance criterion was designed to accelerate convergence and improve accuracy. We named this novel algorithm SESMAM-HH.

The main contributions and innovations of this paper are as follows:

1. For the first time, the effects of multiple models, simultaneous delivery, time windows, and speed regionalization on carbon emissions were considered, and the RLCLRTPTWSPDHF mathematical model was constructed. 
2. The second exponential smoothing method, combined with the adaptive mechanism, was used as the high-level selection strategy of the hyper-heuristic algorithm, and an adaptive receiving mechanism is proposed.

3. The impacts of vehicle composition, depot distribution and cost, and customer parameters on carbon emissions, logistics costs, and customer satisfaction are evaluated, and management advice and guidance on how to plan and manage distribution decisions are provided.

The construction of this paper is as follows: the second section briefly reviews related work; the third section establishes the RLCLRPTWSPDHF integrated three-dimensional exponential model; the fourth section describes the proposed algorithm; the fifth section designs the simulation experiment to verify the correctness of the RLCLRPTWSPDHF and the efficiency of the proposed algorithm, and analyzes the impact of various parameters on logistics costs, carbon emissions, and customer satisfaction; the last section contains the conclusion and outlook.

\section{Review}

LRP integrates two classic NP-hard problems: the location allocation problem (LAP) and the vehicle routing problem (VRP) [3]. At present, LRP has become one of the most important tools and research hotspots for operations management and cargo transportation [4,5]. LCLRP is an LRP problem that aims to reduce greenhouse gases, such as carbon emissions generated in logistics and distribution. It also combines two types of NP-hard problems: LAP and the pollution routing problem (PRP) or green VRP (GVRP). PRP and GVRP focus on the social and environmental impacts of carbon emissions from cargo transportation.

To establish a reasonable model, a model for assessing vehicle carbon emissions is essential. Table 1 lists 14 different fuel consumption/carbon emission models, covering the model classification and source and providing the carbon emission impact factors involved in the model.

Table 1. Fuel consumption/carbon emission model.

\begin{tabular}{|c|c|c|c|c|c|c|c|c|c|c|c|c|c|}
\hline Macro Model & VP & FT & RG & E & $\mathbf{S}$ & AS & Micro Model & VP & FT & RG & E & $\mathrm{S}$ & AS \\
\hline MEET [6] & $\sqrt{ }$ & $\sqrt{ }$ & $\sqrt{ }$ & $\sqrt{ }$ & $\sqrt{ }$ & & IFCM [7] & $\sqrt{ }$ & $\sqrt{ }$ & $\sqrt{ }$ & $\sqrt{ }$ & $\sqrt{ }$ & $\sqrt{ }$ \\
\hline NTM [2] & $\sqrt{ }$ & $\sqrt{ }$ & & & & & FMEFCM [8] & $\sqrt{ }$ & $\sqrt{ }$ & $\sqrt{ }$ & $\sqrt{ }$ & $\sqrt{ }$ & $\sqrt{ }$ \\
\hline COPERT [9] & $\sqrt{ }$ & $\sqrt{ }$ & & & $\sqrt{ }$ & & RSFCM [8] & $\sqrt{ }$ & $\sqrt{ }$ & $\sqrt{ }$ & $\sqrt{ }$ & $\sqrt{ }$ & \\
\hline ECOTRANSIT [10] & $\sqrt{ }$ & $\sqrt{ }$ & $\sqrt{ }$ & $\sqrt{ }$ & $\sqrt{ }$ & $\sqrt{ }$ & ASFCM [11] & $\sqrt{ }$ & $\sqrt{ }$ & $\sqrt{ }$ & $\sqrt{ }$ & & \\
\hline NAEI [12] & $\sqrt{ }$ & $\sqrt{ }$ & & $\sqrt{ }$ & $\sqrt{ }$ & & VSP [13] & $\sqrt{ }$ & $\sqrt{ }$ & $\sqrt{ }$ & $\sqrt{ }$ & $\sqrt{ }$ & $\sqrt{ }$ \\
\hline MOVES [14] & $\sqrt{ }$ & $\sqrt{ }$ & & $\sqrt{ }$ & $\sqrt{ }$ & & OFCM [15] & $\sqrt{ }$ & $\sqrt{ }$ & $\sqrt{ }$ & $\sqrt{ }$ & $\sqrt{ }$ & $\sqrt{ }$ \\
\hline IVE [16] & $\sqrt{ }$ & $\sqrt{ }$ & & & $\sqrt{ }$ & & CMEM [17] & $\sqrt{ }$ & $\sqrt{ }$ & $\sqrt{ }$ & $\sqrt{ }$ & $\sqrt{ }$ & $\sqrt{ }$ \\
\hline
\end{tabular}

In the above table, the meanings of the abbreviation are as follows:

MEET: Methodology for calculating transportation emissions and energy consumption; NTM Network for transport and environment; COPERT: Computer program to calculate emissions from road transportation; ECOTRANSIT: Ecological transport information tool; NAEI: National atmospheric emissions inventory; MOVES: Motor Vehicle Emission Simulator; IVE: The international vehicle emissions model; IFCM: An instantaneous fuel consumption model; FMEFCM: A four-mode elemental fuel consumption model; RSFCM: A running speed fuel consumption model; ASFCM: Average speed fuel consumption model; VSP: Vehicle specific power; OFCM: The Oguchi fuel consumption model; CMEM is the abbreviation of Comprehensive Modal Emissions Modeling. And, VP is the vehicle parameter, FT is the fuel type, RG is the road gradient, E is the environmental factor, $\mathrm{S}$ is the speed, and AS is the acceleration.

The above 14 models involved factors affecting carbon emissions: environmental conditions, vehicle parameters, traffic conditions, and driver factors.

In urban logistics, the use of a heterogeneous fleet (HF) is one of the key factors in reducing logistics costs and carbon emissions and improving logistics distribution efficiency [17-20]. Koç et al. [17,21] 
analyzed the impact of fleet size and composition on costs and carbon emissions. The conclusions showed the advantages of a heterogeneous fleet compared to single-model logistics distribution networks. Li Jin et al. [22] constructed a heterogeneous fleet, low-carbon VRP model with a fixed number of vehicles. The data showed that using HF can improve vehicle capacity utilization and reduce energy consumption and carbon emissions. The number of vehicles can also meet the requirements of actual distribution applications. Pitera et al. [23] studied the role of HF in reverse network recycling, and the results also indicated that the main parameters of $\mathrm{HF}$ reduce $\mathrm{CO}_{2}$ emissions. To reduce $\mathrm{CO}_{2}$ emissions, Xiao et al. [24] considered two types of heavy vehicles with different emission models and analyzed the impact of multi type and homogeneous fleets on $\mathrm{CO}_{2}$ emissions. As a result, they showed that HF has enormous potential to reduce logistics costs and carbon emissions.

Traffic congestion is one of the main factors affecting the emission of greenhouse gases, such as $\mathrm{CO}_{2}$ and fuel conversion efficiency [25]. Barth M. et al. [26] believed that greenhouse gas emissions, such as $\mathrm{CO}_{2}$ and fuel consumption, will increase rapidly and non-linearly when the vehicle speed is below $30 \mathrm{mph}$. For example, when the speed drops from 30 to $12.5 \mathrm{mph}$, or when the speed drops from 12.5 to $5 \mathrm{mph}$, greenhouse gas emissions and fuel consumption per mile of $\mathrm{CO}_{2}$ will double. Therefore, in recent years, many scholars have devoted themselves to analyzing the impact of real-time speed (or uncertainty of travel time) caused by traffic congestion on costs or $\mathrm{CO}_{2}$ emissions. The method adopted is generally to use the segmentation function of speed to simulate traffic congestion [24,27-33]. In addition, Poothalir et al. [34] used a triangular probability distribution function curve to characterize the time-varying velocity and found that the change in velocity is beneficial for reducing fuel consumption. Scholars interested in the time-varying rate of traffic congestion can refer to the literature [35].

\section{The Model of RLCLRPTWSPDHF}

\subsection{Fuel Consumption/Carbon Emission Model}

This section introduces the fuel consumption, carbon emission model, namely, the comprehensive modal emission model (CMEM). At present, the CMEM model has been applied to PRP and its variants $[5,7,8,11-13,34,36]$. The CMEM model is shown in Equation (1):

$$
F R^{h}=\xi\left(k^{h} N^{h} V^{h}+P^{h} / \eta\right) / k,
$$

where $\xi$ is the ratio of fuel to air mass; $k^{h}, N^{h}$ and $V^{h}$ are the engine friction coefficient, speed, and displacement, respectively, of vehicle $h ; \eta$ is the diesel/gasoline efficiency value; $k$ is the diesel/gasoline calorific value; and $P^{h}$ is the engine power output $(\mathrm{KW} / \mathrm{s})$ of vehicle $h$. Its expression is shown in Equation (2):

$$
P^{h}=\frac{P_{\text {tract }}^{h}}{n_{t f}}+P_{a c c}
$$

where $n_{t f}$ is the efficiency of the vehicle transmission system; $P_{a c c}$ is associated with engine running loss and vehicle accessories, such as air conditioning and electric load, and is usually set to 0 ; and $P_{\text {tract }}^{h}$ is the total traction power (KW/s) of vehicle $h$. Its expression is shown in Equation (3):

$$
P_{\text {tract }}^{h}=\left(M^{h} \tau+M^{h} g \sin \theta+0.5 C_{d}^{h} \rho A^{h} v^{2}+M^{h} g C_{r} \cos \theta\right) v / 1000,
$$

where $M^{h}$ is vehicle mass $(\mathrm{kg}) ; \tau$ is acceleration $\left(\mathrm{m} / \mathrm{s}^{2}\right) ; g$ is gravity acceleration $\left(\mathrm{m} / \mathrm{s}^{2}\right) ; \theta$ is slope; $C_{d}^{h}$ is the air resistance coefficient of vehicle $h ; A^{h}$ is the windshield area $\left(\mathrm{m}^{2}\right)$ of vehicle $h$; $v$ is the vehicle speed $(\mathrm{m} / \mathrm{s})$; and $C_{r}$ is the rolling resistance coefficient.

If vehicle $h$ carries weight $M^{h}$, then the amount of fuel consumed when travelling at speed $v$ (constant) over a distance $d(\mathrm{~m}), F^{h}(\mathrm{~L})$, is $F^{h}=F R^{h} \times t(t$ is time, unit: s), i.e.,

$$
F^{h}=\left(\frac{\xi k^{h} N^{h} V^{h}}{\kappa \psi}+\frac{\xi P^{h}}{\eta \kappa \psi}\right) \times \frac{d}{v} .
$$


Let $\lambda=\xi / k \psi$ ( $\psi$ is the conversion factor), $\gamma=\frac{1}{1000} n_{t f} \eta, \beta^{h}=0.5 C_{d}^{h} A^{h}$ and $\alpha=\tau+g \sin \theta+g C_{r} \cos \theta$. Then, Equation (4) is further simplified:

$$
F^{h}=\lambda\left(k^{h} N^{h} V^{h} d / v+M^{h} \gamma \alpha d+\beta^{h} \gamma d v^{2}\right)
$$

where $k^{h} N^{h} V^{h} d / v$ is the engine module, which is proportional to the running time; $M^{h} \gamma \alpha d$ is the load module, which is proportional to the mass and distance of the whole vehicle; and $\beta^{h} \gamma d v^{2}$ is the speed module, which is proportional to the square of the distance and speed. Figure 1 shows the relationship between fuel consumption and speed when the vehicle travels $100 \mathrm{~km}$. Figure 1a shows the relationship between the fuel consumption and the vehicle speed when the vehicle's own weight is not loaded, and Figure $1 \mathrm{~b}$ shows the relationship between the fuel consumption and the vehicle speed when the vehicle's own weight is loaded.

In the Figure, L1, L2, and M represent three vehicles with different parameters. It can be seen from Figure 1 that the relationship between fuel consumption and velocity exhibits a U-shaped curve and the optimal velocity exists to minimize fuel consumption, that is, $v_{\text {best }}=\left(k^{h} N^{h} V^{h} / 2 \beta^{h} \gamma\right)^{1 / 3}$. The $\mathrm{CO}_{2}$ emissions of vehicles are linearly proportional to fuel consumption; the larger the fuel consumption is, the larger the carbon emission. The conversion coefficient $\eta_{T}$ of gasoline and carbon dioxide is 2.32 [17], that is, $2.32 \mathrm{~kg}$ of $\mathrm{CO}_{2}$ is produced for every $1 \mathrm{~L}$ of gasoline consumed.
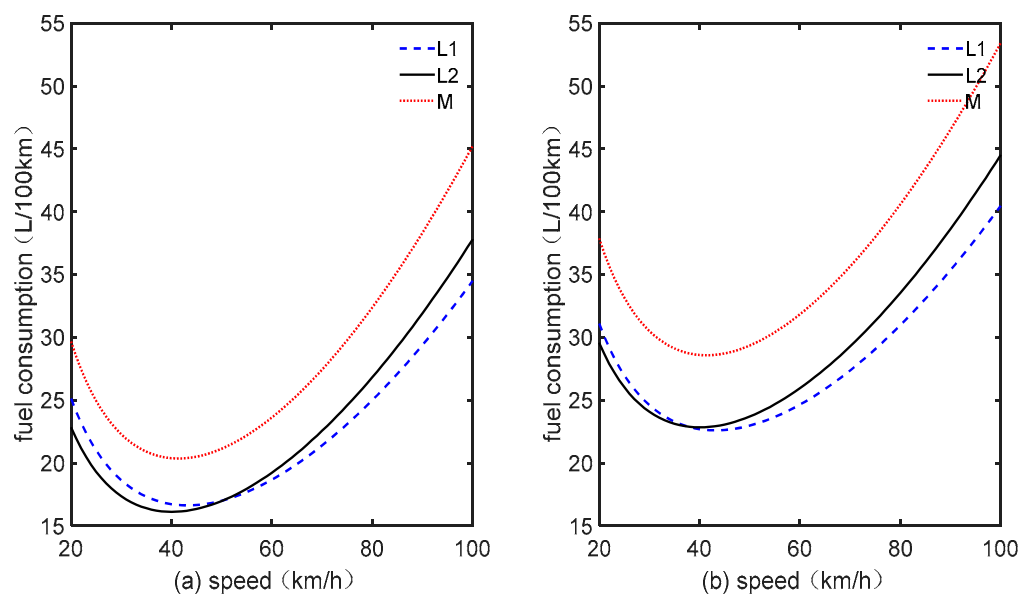

Figure 1. Fuel consumption as a function of speed.

\subsection{Speed Regionalization}

Speed limits help to reduce driving, save fuel, and reduce the emission of pollutants [37]. The regionalization of speed reflects the speed and time variation caused by traffic congestion, which can effectively alleviate traffic congestion and achieve the sustainable development of traffic. Figure 2 is a simple and effective way to regionalize speed. The city is divided into three speed areas: Zone 1 corresponds to the central belt, which is embodied in lanes and narrow roads; Zone 2 corresponds to the business district and university city outside the city center; and Zone 3 corresponds to the suburbs, including agricultural areas and parks. $V_{\text {zone } 1}, V_{\text {zone2 }}$, and $V_{\text {zone } 3}$ indicate the fixed driving speed of each area, where $V_{\text {zone } 1}<V_{\text {zone } 2}<V_{\text {zone3 }}$. When the vehicle is travelling in the same area, its speed is equal to the speed of the area. When traveling on the boundary of the two speed zones, the vehicle travels at a speed that makes the value of $K=k^{h} N^{h} V^{h} / v+\beta^{h} \gamma v^{2}$ smaller.

In this paper, the driving speed of each area was randomized. We set $V_{\text {zone } 3} \sim \mathrm{U}(60,80) \mathrm{km} / \mathrm{h}$, $V_{\text {zone } 2} \sim \mathrm{U}(40,60) \mathrm{km} / \mathrm{h}$, and $V_{\text {zone } 1} \sim \mathrm{U}(20,40) \mathrm{km} / \mathrm{h}$. In addition, the strategy of [17] was used to calculate the non-dominated driving route between any two nodes, and the carbon emissions were calculated based on the load between the two nodes. 


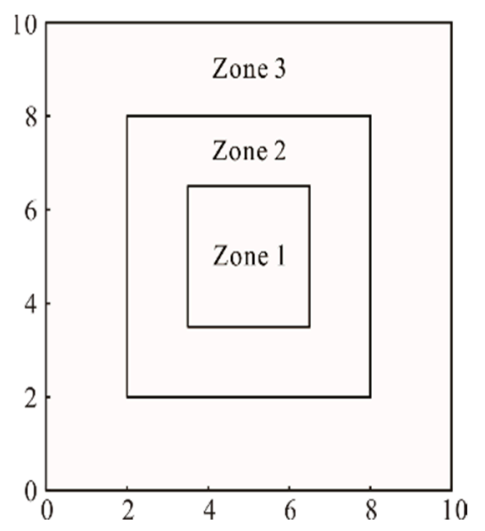

Figure 2. Illustration of speed zones.

\subsection{Mathematical Model}

RLCLRPTWSPDHF can be defined as a complete graph $G=(V, E)$, where $V$ is composed of candidate depots $M$ and $N$ customers. Each customer has demand and time windows, with known geographical locations. The capacity, location, and open cost of each candidate depot are known. $E$ is the edge set. Different types of vehicles depart from the depot to serve a series of customers and return to the original starting point. The goal of the model was to minimize total costs, including the fixed open costs of depots, vehicles costs, fuel and carbon emissions costs, and penalty costs.

The model was based on the following assumptions: (1) every customer is served exactly once; (2) the vehicle must return to the original depot after serving all customers; (3) the vehicle capacity is not exceeded; (4) the depot capacity is not exceeded; and (5) each depot has enough vehicles to serve the corresponding customers.

The meanings of the symbols and variables that appear in the model are as follows: customer nodes $I=\{1,2, \ldots, N\}$, potential depot nodes $J=\{N+1, N+2, \ldots, N+M\}$, heterogeneous fleet $H=\{L 1, L 2, M\}$, the set of nodes $V=I \cup J$, and the $\operatorname{arcset} E=\{(i, j): i, j \in V, i \neq j\} \backslash\{(i, j): i \in J, j \in J\}$. $F D_{j}$ and $C D_{j}$ are the open costs and capacities of the potential depots, respectively. $p_{i}$ and $q_{i}$ are the pickup and delivery demand of customer $i .\left[e_{i}, l_{i}\right]$ is the soft time window of customer $i . S T_{i}$ is the service time for customer i. $C V_{h}$ and $F V_{h}$ are the capacity and fixed dispatch cost, respectively, of vehicle $h . A T_{i h}$ is the time when the vehicle arrives to the customer $i . T T_{i j h}$ is the travel time of vehicle $h$ in $\operatorname{arc}(i, j) . Q_{i j h}$ is the dynamic load of vehicle $h$ on $\operatorname{arc}(i, j) . F_{i j h}$ is the fuel consumption of vehicle $h$ on $\operatorname{arc}(i, j) . C_{f}$ is the fuel consumption cost (yuan/liter). $C_{c o 2}$ is the cost of carbon emissions (yuan $/ \mathrm{kg}$ ), and $C_{v w} / C_{c w}$ is the early or late penalty coefficient of the vehicle (yuan/min).

The decision variables are as follows: $x_{i j h}$ indicates whether arc $(i, j)$ is served by vehicle $h$. If it is, $x_{i j h}$ takes the value of 1 ; otherwise, $x_{i j h}$ is $0 . z_{i j}$ indicates whether client $i$ is served by candidate depot $j$. If it is, then $z_{i j}$ takes the value of 1 ; otherwise, it is $0 . y_{j}$ indicates whether candidate depot $j$ is leased. If it is, $y_{j}$ takes the value of 1 ; otherwise, it is 0 . Based on the above assumptions and definitions, the model is constructed as follows:

$$
\begin{gathered}
\min C=\sum_{j \in J} F D_{j} y_{j}+\sum_{i \in J} \sum_{j \in I} \sum_{h \in H} F V_{h} x_{i j h}+\left(C_{f}+C_{c 0_{2}} \eta_{T}\right) \sum_{i \in V} \sum_{j \in V} \sum_{h \in H} F_{i j h} x_{i j h}+C_{v w} \sum_{i \in I} \sum_{j \in V} \sum_{h \in H} x_{i j h} \max \left\{e_{i}-A T_{i h}, 0\right\}+ \\
C_{c w} \sum_{i \in I} \sum_{j \in V} \sum_{h \in H} x_{i j h} \max \left\{\max \left\{A T_{i h}, e_{i}\right\}+S T_{i}-l_{i}, 0\right\} ; \\
\sum_{i \in V} \sum_{h \in H} x_{i j h}=1, \forall j \in I ; \\
\sum_{h \in H} \sum_{i \in V} x_{i j h}=\sum_{h \in H} \sum_{i \in V} x_{j i h}, \forall j \in V ; \\
\sum_{j \in J} z_{i j}=1, \forall i \in I ;
\end{gathered}
$$




$$
\begin{gathered}
x_{i j h}+\sum_{b \in H, b \neq h} \sum_{r \in V, r \neq j} x_{j r b} \leq 1, \forall i \in V, j \in I, i \neq j, h \in H ; \\
\sum_{h \in H} x_{i j h} \leq z_{i j}, \forall i \in I, j \in J ; \\
\sum_{h \in H} x_{j i h} \leq z_{i j}, \forall i \in I, j \in J ; \\
\sum_{h \in H} x_{i j h}+z_{i k}+\sum_{m \in J, m \neq k} z_{j m} \leq 2, \forall i, j \in I, k \in J, i \neq j ; \\
\max \left\{\sum_{i \in I} q_{i} z_{i k}, \sum_{i \in I} p_{i} z_{i k}\right\} \leq C D_{k} y_{k}, \forall k \in J ; \\
\sum_{i \in J} \sum_{j \in I} Q_{i j h}=\sum_{i \in I} \sum_{j \in V} q_{i} x_{i j h}, \forall h \in H ; \\
\sum_{i \in I} \sum_{j \in J} Q_{i j h}=\sum_{i \in I} \sum_{j \in V} p_{i} x_{i j h}, \forall h \in H ; \\
\sum_{i \in V} \sum_{h \in H} x_{i j h}\left(Q_{i j h}-q_{j}\right)=\sum_{i \in V} \sum_{h \in H} x_{j i h}\left(Q_{j i h}-p_{j}\right), \forall j \in I ; \\
Q_{i j h} \leq C V_{h} x_{i j h}, \forall i, j \in V, i \neq j, h \in H ; \\
Q_{i j h} \geq\left(q_{j}-p_{j}\right) x_{i j h}, \forall i \in V, j \in I, h \in H ; \\
z_{j h}=x_{i j h} \cdot\left(\max \left\{e_{i}, A T_{i h}\right\}+S T_{i}+T T_{i j h}\right), \forall i, j \in V, h \in H ; \\
x_{i j h} \in\{0,1\}, \forall i, j \in V, h \in H ; \\
y_{j} \in\{0,1\}, \forall j \in J ;
\end{gathered}
$$

where Equation (6) is the objective function, including the dispatch costs of the vehicles and depots, fuel consumption and carbon emission costs, and the vehicles' early or late penalty costs; Equations (7) and (8) guarantee that each customer can only be served once; Equations (9) and (10) guarantee that each customer can only be serviced by one depot and one vehicle; Equations (11)-(13) ensure that the service vehicle returns to the original depot; Formula (14) is the depot capacity constraint; Equations (15) and (16) are the load at the time of departure and return of the vehicle; Equation (17) is the dynamic balance constraint of the vehicle load; Equations (18) and (19) are the vehicle capacity constraints; Equation (20) is the time constraint to reach the customer; and Equations (21)-(23) are decision variables.

When the fuel consumption and carbon emission costs in Equation (6) are replaced by the distance cost, RLCLRPTWSPDHF is changed to the Regional LRP, considering time windows, simultaneous pickup, and delivery with heterogeneous fleets (RLRPTWSPDHF).

\section{Algorithm}

The hyper-heuristic (HH) algorithm system was proposed by Cowling et al. [38] in 2000 and is defined as a "heuristic selection heuristic" algorithm. Later, Burke [39] and others expanded its definition to include heuristic selection and heuristic generation. In the framework of the $\mathrm{HH}$, the domain barrier is used to isolate the high-level heuristic (HLH) and the low-level heuristic (LLH). The low-level problem domain contains a series of low-level operators, problem definitions, objective functions, and other information. There are two different target strategies in the high-level control domain: the selection strategy of the operator and the receiving mechanism of the solution. 
The selection strategy is used to monitor the performance information of the LLH to select a good and suitable operator (separating any information related to the actual problem). The receiving mechanism determines whether to replace the parent solution according to the quality of the child solution and controls the search direction of the algorithm and the convergence speed.

In addition, there is a message transmitter between the high-level domain and the underlying problem domain, which is used to transmit information that is not related to the problem domain, including selection information, judgement information about the receiving strategy, improvement rates provided by the underlying layer, operator running time and number of times, number of consecutive unimproved solutions for the current solution, and the like.

RLCLRPTWSPDHF is an NP problem. This paper designed an SESMAM-HH to solve this problem. We have designed a series of low-level operators that conform to this problem, and a high-level selection strategy and receiving mechanism with a certain general level. The purpose was to exploit the portability and robustness of the $\mathrm{HH}$ algorithm for other areas of research and reduce the difficulty of designing the underlying algorithm.

RLCLRPTWSPDHF and the proposed algorithm SESMAM-HH were studied according to the following four steps: (1) coding the solution and constructing the initial population; (2) designing the $\mathrm{HLH}$, including the adaptive selection mechanism and the adaptive receiving mechanism based on secondary exponential smoothing; (3) designing the LLH pool of operators $\xi$, including six local search operators (local search, LS) and nine mutational operators (mutational heuristic, $\mathrm{MH}$ ); and (4) analyzing the performance of the $\mathrm{HH}$.

\subsection{Coding and Initial Population Generation}

In the problem domain, a complete solution can be represented as a collection of all routes, that is $R=\left\{r_{1}, r_{2}, \ldots r\right\}$. The first and last stops of each vehicle travelling route $r_{i}$ are selected depots, the middle part is the customer node number, indicating the access order, and each route is stored in the corresponding cell array. The chromosome length is a non-fixed-length natural sequence related only to the number of vehicles, $K$, and the size, $N$, of the customer, and the value is $2 K+N$. The length of the cell arrays is $K$ (the number of vehicles is variable). To calculate the fitness quickly, the attributes of each vehicle's driving route are also included in $R$ (stored in the cell array in the second row), which is defined as the route attribute line, including the starting load of the vehicle, route travel cost and vehicle type. When calculating the fitness value, we only need to call the cost generated by the route in the attribute line, avoiding unnecessary double counting. Figure 3 is a simple example of a customer set: $I \in\{1,2, \ldots, 15\}$, depot set $J \in\{16,17,18,19\}$. Similar to the hierarchical tree coding method [6], the root system is the driving route, which includes four routes; the branches are the number of vehicles, including four service vehicles; and the leaves are the attributes of the route, including the starting and stopping load, the route cost, and the vehicle type.

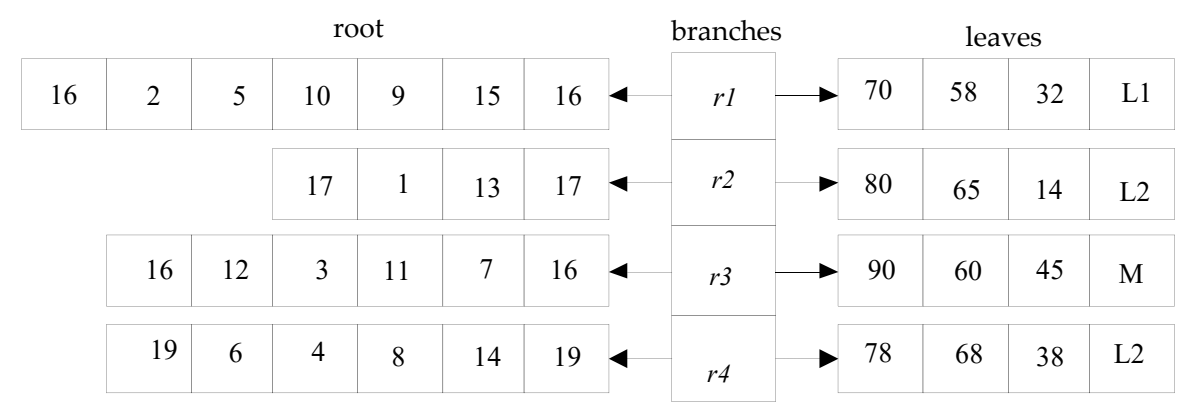

Figure 3. Illustration of chromosome representation.

The SESMAM-HH adopts single-point search framework (SPSF). To avoid the disadvantage that SPSF can easily fall into the local solution, each independently running individual is randomly selected from the initial population. First, the customer sequence is randomly generated, the customer is 
assigned, and the vehicle is dispatched using the greedy rule under the condition that the constraint is satisfied. Second, according to the vehicle route that is formed, the depot is randomly selected (the capacity constraint of the depot needs to be satisfied), the attributes of each route are calculated, and finally the complete vehicle route set is constructed.

\subsection{High-level Strategy Design}

\subsubsection{Selection Strategy Design}

$\mathrm{HH}$ is a hotspot in many combinatorial optimization fields, and many excellent selection strategies have emerged, including Choice Function (CF) [38] and Fitness Rate Rank based Multi-Armed Bandit (FRR-MAB) [40]. This paper proposes a novel selection strategy, namely, SESMAM based on the second exponential smoothing method combined adaptive mechanism, which has the following three characteristics:

(1) This method uses the second exponential smoothing method to predict the performance of LLH; (2) according to the global historical performance, the LLH operators are divided into elite heuristics $(\mathrm{EH})$ and poor heuristics $(\mathrm{PH})$, and select the appropriate $\mathrm{EH}$ or $\mathrm{PH}$ according to the performance of the algorithm in the recent stage; and (3) this paper designs different performance evaluation methods for $\mathrm{EH} / \mathrm{PH}$.

According to Single Exponential Smoothing (SES):

$$
S_{i, t}(1)=\alpha \cdot F I R_{i, t}+(1-\alpha) \cdot S_{i, t-1}(1),
$$

where $S_{i, t}(1)$ is an exponential smoothing value when the operator $i \in \xi$ is called at the th period and $\alpha$ is the smoothing coefficient; $F I R_{i, t}$ is the fitness improvement rate of the operator $i(i \in \xi)$. When the initial value $S_{i, 0}(1)$ is set to zero, then Equation (24) may be written as Formula (25):

$$
S_{i, t}(1)=\alpha \sum_{r=0}^{t}(1-\alpha)^{t-r} F I R_{i, r}+(1-\alpha)^{t} S_{i, 0}(1)
$$

The SES method has the advantages of simplicity and fast operation, but when the variation in the sample sequence has a tendency to approximate a straight line, using SES for prediction will cause hysteresis deviation. Therefore, the smoothing process is usually performed again on a smooth basis, which enables a more accurate prediction, as follows:

$$
\begin{gathered}
S_{i, t}(2)=\alpha \cdot S_{i, t}(1)+(1-\alpha) \cdot S_{i, t-1}(2) ; \\
Y_{i, t+1}=a_{t}+b_{t} ; \\
\left\{\begin{array}{l}
a_{t}=2 S_{i, t}(1)-S_{i, t}(2) \\
b_{t}=\frac{\alpha}{1-\alpha}\left(S_{i, t}(1)-S_{i, t}(2)\right)
\end{array} ;\right.
\end{gathered}
$$

where $S_{i, t}(2)$ is the second exponential smoothing value of the operator $i(i \in \xi)$ at the $t$ th period and $Y_{i, t+1}$ is the performance prediction value of the operator $i(i \in \xi)$ at the $(t+1)$ th period.

$\xi$ can generally be divided into two categories, $\mathrm{EH}$ and $\mathrm{PH}$, which can be classified using the global historical performance GFIR (Global FIR) of the operator, as shown in the following equation:

$$
\operatorname{GFIR}(i)=\operatorname{GFIR}(i)+F I R_{i, t}
$$

If $\operatorname{GFIR}(i)$ is greater than or equal to 0 , then the operator $i$ is classified as $\mathrm{EH}$; otherwise, it is $\mathrm{PH}$. If all the values of GFIR are less than 0, the GFIR are arranged in descending order, taking the bottom operator of the first $1 / 2$ as EH and the rest as $\mathrm{PH}$. During the iteration, an adaptive selection mechanism 
is proposed, that is, the probability of selecting PH increases with the increase in TQ (see Equation (30)). $\mathrm{TQ}$ is the number of uses of $\mathrm{EH}$ without improving the current solution.

$$
p_{P H}=\left(\frac{2 \times T Q}{N T}\right)^{\varphi} .
$$

In Equation (30) $\varphi$ is the weight and controls the selection probability and NT is the total number of operators. At the same time, it is not reasonable to use FIR as the performance of the PH operator. Therefore, this paper takes the improvement rate of the global optimal solution obtained by two consecutive calls of $\mathrm{PH}$ operator as the performance of $\mathrm{PH}$ operator. The calculation formula is shown in Formula (31):

$$
\operatorname{PFIR}(i)=\operatorname{PFIR}(i)+\left(\frac{t}{T_{\max }}\right)^{2} \cdot \frac{G F_{t+1}-G F_{t}}{G F_{t}},
$$

where $\operatorname{GFIR}(i)$ is the global historical performance of the operator $i(i \in P H) ; G F_{t}$ is the global optimal solution obtained by calling operator $i(i \in P H)$ at the $t$ th period; $G F_{t+1}$ is the global optimal solution obtained by calling operator $i(i \in P H)$ at the $(t+1)$ th period. Operator credit value is allocated by the FRR-MAB method, as shown in Equation (32):

$$
C V_{i, t}=\frac{\sum_{i t e r=1}^{t} P F_{i, t}}{\sum_{i \in \xi} \sum_{\text {iter }}^{t} P F_{i, t}}+C \sqrt{\frac{2 \times \ln \sum_{i \in \xi} N_{i}^{t}}{N_{i}^{t}}}
$$

where $C V_{i, t}$ is the credit value of operator $i$; $N_{i}^{t}$ is the number of times operator $i$ is used in t iterations; and $C$ is the balance factor of exploration and development, which is used to prevent the singularity of operator selection; $P F_{i, t}$ is the performance value of the operator, and when the $\mathrm{PH}$ operator is selected, it equals PFIR; when the EH operator is selected, it equals $Y$.

Finally, using a roulette to convert performance credits into selection probabilities ensures the diversity of the algorithm in the selection process. The flow chart of the SESMAM method is shown in Figure 4:

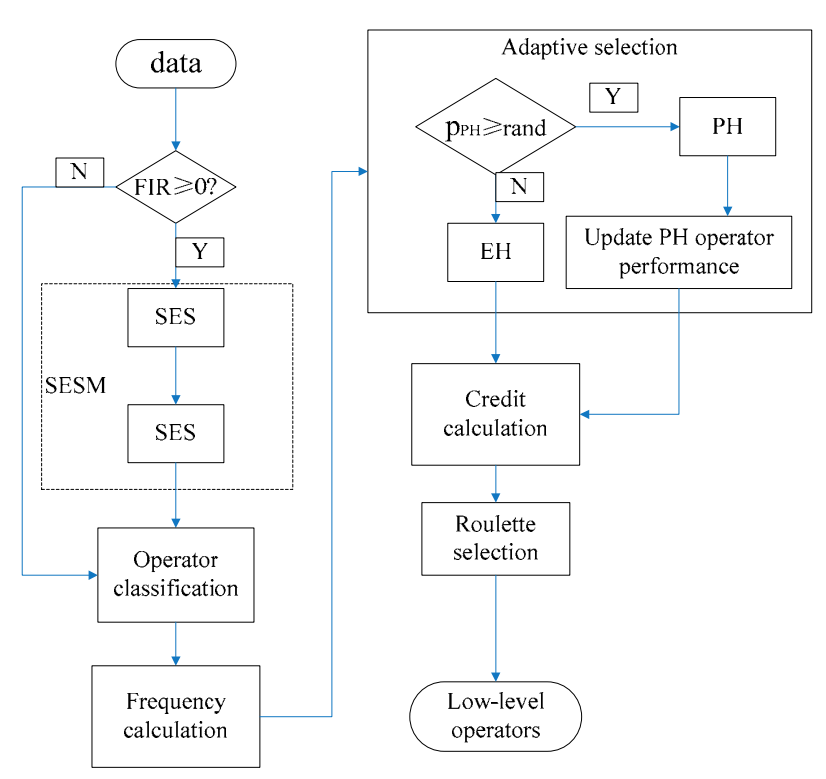

Figure 4. Framework of the second exponential smoothing (SESMAM). 


\subsubsection{Acceptance Criterion Design}

The acceptance criterion (AC), which is used to determine whether the child solution $c f$ replaces the parent solution $p f$, directly affects the convergence speed and optimization accuracy of the hyper-heuristic algorithm. The general AC only uses the improvement rate information and ignores the stage performance information of the algorithm (the operator performance is inconsistent in different optimization stages). In this paper, a simple adaptive AC was constructed by using the stage performance information of the algorithm, which is defined as adaptive acceptance (AA), as shown in Equation (33). If the selected operator improves the current solution, then reset $T Q$ to 0 or infinity. This method is defined as adaptive acceptance (AA), as in Equation (33):

$$
p_{A C}=\left(\frac{2 \times T Q}{N T}\right)^{\psi}
$$

where $\psi$ is the receiving factor and controls the probability of acceptance.

\subsection{Low-Level Operator Design}

The operator library, $\xi$, can be directly used to search the problem domain solution space, provided by problem domain experts. $\xi$ can be regarded as an inoperable black box, divided into two categories: local search (LS) and mutation heuristic (MH). $\mathrm{MH}$ has a small perturbation to the current solution to prevent it from falling into a local optimal solution. The disadvantage is that a high-quality solution cannot be guaranteed. However, LS is used to improve the current solution and obtain a high-quality solution. This paper constructed six LS operators and nine MH operators.

LS includes: 2-Opt inside one route (Inside-2Opt), 2-Opt between different routes (Inter-2Opt), swapping customers inside one route (Inside-Swap), swapping customers between different routes (Inter-Swap), shifting customers inside one route (Inside-Shift) and shifting customers inter different routes (Inter-Shift). For local optimization inside one route, a full optimization strategy is used [41]. Local optimization between different routes uses the K-1 optimization mechanism; that is, a route is randomly selected, and optimized with customers from other routes. Therefore, the operator complexity is reduced from $K(K-1) / 2$ to $K-1$.

$\mathrm{MH}$ was divided into two categories, one for vehicle route disturbances only, including Inside-2Opt-M, Inside-Or-Opt, Inter-Shift-M, Inter-Swap-M, Shaw [36], Decompose, and Merge. The strategy of the first five perturbation operators is to randomly select the $1 / 4 \sim 1 / 2$ route and randomly perturb the customers among the lines; the Decompose method involves decomposing a randomly selected route into two routes; Merge randomly chooses two routes and merges them into one. The other type of $\mathrm{MH}$ perturbs the selected warehouse, including Add-Swap and Relocation. The Add-Swap operator randomly selects a $1 / 3$ to $2 / 3$ route and allocates it to a new depot or closes a depot and arranges all the routes of this depot to another depot to prevent excessive convergence due to there being too few depots. Relocation collapses each driving route into a "Super-Client", and then inserts the depot into each route to obtain a minimum insertion cost [42].

The above two types of operators must satisfy the constraint (7)-(20) to ensure the feasibility of the route and to avoid the use of repair techniques, and the LS operator must guarantee FIR $\geq 0$.

\subsection{Algorithm Complexity Analysis}

The time complexity of an algorithm is a relative measure of runtime that is used to measure the runtime performance of an algorithm. The complexity of an $\mathrm{HH}$ iteration can be divided into four parts according to the $\mathrm{HH}$ process framework: high-level selection strategy complexity, low-level operator execution complexity, high-level solution acceptance complexity, and elite retention strategy complexity.

For the single-point search algorithm framework, when the number of iterations is $T_{\max }$, the number of customers is $N$, the number of vehicles is $K$, and the number of low-level operators is $N L$, and the algorithmic iteration complexity is calculated as follows: The complexity of the high-level selection 
strategy is $O(H S)$, the maximum complexity of the low-level operator is $O\left(N^{2}\right)$, the maximum complexity of the high-level solution acceptance mechanism is $O(4)$, and the complexity of the elite retention strategy is $O(2)$. There is also the population initialization complexity $N_{p o p} \times O\left(N^{2}\right)$ and the fitness calculation complexity $N_{p o p} \times O\left(N^{2}\right)$ ( $N_{p o p}$ is the initial population size), so the overall complexity of the algorithm is $O(H H)$ :

$$
O(H H)=2 N_{\text {pop }} \times O\left(N^{2}\right)+T_{\max } \times\left(O(H S)+O\left(N^{2}\right)+O(6)\right),
$$

where $O(H S)$ includes two SESM computational complexities $O(5)$, the low-level operator classification complexity $O(N L+1)$, the adaptive probability computational complexity $O(2)$, $\mathrm{PH}$ performance computational complexity $O(1)$, credit value computational complexity $O(N L)$, and the roulette strategy complexity $2 O(N L)$; that is:

$$
\begin{gathered}
O(H S)=4 \times O(N L)+O(9) ; \\
O(H H)=2 N_{p o p} \times O\left(N^{2}\right)+T_{\max } \times O\left(N^{2}\right)+4 T_{\max } \times O(N L)+T_{\max } \times O(15) .
\end{gathered}
$$

Therefore, the overall computational complexity of the algorithm is $T_{\max } \times O\left(N^{2}\right)$, indicating that the $\mathrm{HH}$ algorithm is an effective algorithm.

\section{Simulation Experiment and Analysis}

The purpose of the simulation experiment was as follows: (1) verify the accuracy of the algorithm and the correctness of the model; (2) verify the efficiency of the proposed SESMAM-HH; and (3) analyze the impact of various parameters such as time windows, distribution center distribution, and vehicle type on cost, carbon emissions, and customer satisfaction.

The proposed algorithm used MATLAB 2015b parallel programming. The computer environment was an Intel(R) Core(TM) CPU i7-6700K@4.00 GHz, 8 GB RAM, and Windows 10. The termination condition was $T_{\max }=\min \left(10 \times(M+N+K)^{2}, 10^{5}\right)$. Individuals running independently $(O I=20$ times $)$ were randomly selected from the initial population $\left(N_{p o p}=10\right)$. The other key parameters of the algorithm were the smoothing coefficient, $\alpha \in[0.4,0.6]$; balance factor, $C \in[0.4,0.6]$; control parameter, $\phi \in[0.8,1.6]$; and receiving factor, $\psi \in[2.2,2.4]$. In the following experiment, the above four parameters were randomly selected from the optimal range.

\subsection{Analysis of Model Correctness and Algorithm Performance}

There are no benchmarks for RLCLRPTWSPDHF, and the following examples are arranged according to the purpose of the experiment -15 simple examples were randomly generated, satisfying the following conditions: customer number $N \in\{10,15,20\}$; depot number $M \in\{2,3,5\}$; customer pickup quantity $q$ and delivery quantity $p$ obeyed $U\{100,1100\}(\mathrm{kg})$; the customer time window was randomly generated and guaranteed $l_{i}-e_{i} \geq S T_{i}(S T \in U\{0,10\}$ (minutes)); the vehicle type with different capacities was $\{L 1, L 2, M\}$ fixed vehicle costs were $F V \in\{37.18,43.38,53.11\}$ (yuan); depot capacity was $C D \in\left\{104,1.5 \times 10^{4}\right\}(\mathrm{kg})$; single depot costs, FD, were 200 yuan (Zone 3), 350 yuan (Zone 2), and 500 yuan (Zone 1 ); $C_{v w}=0.3$ yuan $/$ minute; $C_{c w}=0.5$ yuan $/ \mathrm{minute} ; C_{f}=7.60$ yuan $/ \mathrm{minute}$; and $C_{c o 2}=38.49$ yuan/ton (Shanghai transaction price on 4 May 2018); Other data can be referred to in [17].

To verify the correctness of the proposed model and the accuracy of the algorithm, the 15 examples above were solved by SESMAM-HH and CPL-EX. The results are shown in Table 2. In the table, the first column is the example name $L N \times M-N o$., $\mathrm{N}$ and $\mathrm{M}$ are the number of customers and depots, No. indicates the serial number at the same scale, TC is the total cost (yuan), Gap is the percentage between the two TCs, SD is the standard deviation of the total cost of five independent runs, MT is the average running time (s) of five independent runs, and $\mathrm{Av}$. is the average. 
The results show that the proposed algorithm, SESMAM-HH, can find the optimal solution within a reasonable calculation time and ensure that the standard deviation of all instances is zero. Therefore, it can be proven that the proposed model is correct, and that the accuracy of the algorithm is high.

Table 2. Results of small instances.

\begin{tabular}{|c|c|c|c|c|c|c|c|c|c|c|c|}
\hline \multirow{2}{*}{ Instance } & \multicolumn{2}{|c|}{ CPLEX } & \multicolumn{3}{|c|}{ HH-SESMAM-AA } & \multirow{2}{*}{ Instance } & \multicolumn{2}{|c|}{ CPLEX } & \multicolumn{3}{|c|}{ HH-SESMAM-AA } \\
\hline & TC & MT & Gap & SD & MT & & TC & MT & Gap & SD & MT \\
\hline $\mathrm{L} 10 \times 2-1$ & 441.54 & 3.61 & 0.0 & 0.0 & 0.37 & $\mathrm{~L} 15 \times 3-4$ & 461.54 & 5.59 & 0.0 & 0.0 & 1.53 \\
\hline $\mathrm{L} 10 \times 2-2$ & 425.41 & 2.15 & 0.0 & 0.0 & 0.35 & $\mathrm{~L} 15 \times 3-5$ & 472.86 & 12.64 & 0.0 & 0.0 & 1.59 \\
\hline $\mathrm{L} 10 \times 2-3$ & 529.95 & 0.87 & 0.0 & 0.0 & 0.37 & $\mathrm{~L} 20 \times 5-1$ & 788.76 & 890.45 & 0.0 & 0.0 & 3.74 \\
\hline $\mathrm{L} 10 \times 2-4$ & 549.78 & 0.65 & 0.0 & 0.0 & 0.36 & $\mathrm{~L} 20 \times 5-2$ & 894.56 & 501.18 & 0.0 & 0.0 & 3.46 \\
\hline $\mathrm{L} 10 \times 2-5$ & 424.88 & 1.12 & 0.0 & 0.0 & 0.39 & $\mathrm{~L} 20 \times 5-3$ & 746.06 & 1061.27 & 0.0 & 0.0 & 3.75 \\
\hline $\mathrm{L} 15 \times 3-1$ & 484.02 & 15.37 & 0.0 & 0.0 & 1.37 & $\mathrm{~L} 20 \times 5-4$ & 950.89 & 1667.13 & 0.0 & 0.0 & 3.84 \\
\hline $\mathrm{L} 15 \times 3-2$ & 638.62 & 10.26 & 0.0 & 0.0 & 1.39 & $\mathrm{~L} 20 \times 5-5$ & 916.30 & 476.24 & 0.0 & 0.0 & 3.53 \\
\hline \multirow[t]{2}{*}{$\mathrm{L} 15 \times 3-3$} & 545.40 & 7.94 & 0.0 & 0.0 & 1.64 & & & & & & \\
\hline & & & & & & Av. & 618.04 & 310.43 & 0.0 & 0.0 & 1.85 \\
\hline
\end{tabular}

\subsection{Efficient Analysis of High-Level Strategies}

Experiments in this section used the Barreto [42] example for LCLRP and the $W$ class of the CLAM and YLBAM instance library [43] for the capacitated location-routing problem with simultaneous pickup and delivery (CLRPSPD). The $\mathrm{N}$ in the example was between 21 and 150, and the $\mathrm{M}$ was between 5 and 15 .

The experimental results are shown in Table 3, where the BKS is the best-known solution, Gap1 and Gap2 are the optimal solution and the percentage difference between the average and the BKS, SD and MT are the optimal solution standard deviation and average running time (seconds). The average value, Av., and the median value, Md., are given at the end (the black body indicates that the number is equal to the BKS, black body and italic indicate new BKS). This section sets $T_{\max }=\min \left(5 \times(M+N+K)^{2}, 8 \times 10^{4}\right)$, and the number of independent running is $O I=10$.

It can be seen from Table 3 that SESMAM-HH obtained the optimal solution for all LCLRP examples, and the optimal solution for 12 CLRPSPD examples. Three new BKSs were obtained with an improvement rate of $0.8 \%$ o, $6.7 \%$, and $4.4 \%$. This experiment proves the effectiveness of the proposed algorithm in solving the CLRPSPD/LCLRP.

Table 3. Quality of results by proposed approach for LCLRP and CLRPSPD.

\begin{tabular}{|c|c|c|c|c|c|c|c|c|c|c|}
\hline \multirow{2}{*}{ Instance } & \multirow{2}{*}{ BKS [44] } & \multicolumn{4}{|c|}{ LCLRP } & \multirow{2}{*}{ BKS [43] } & \multicolumn{4}{|c|}{ CLRPSPD } \\
\hline & & $\mathrm{Gap}_{1}$ & $\mathrm{Gap}_{2}$ & SD & MT & & $\mathrm{Gap}_{1}$ & $\mathrm{Gap}_{2}$ & SD & MT \\
\hline $\mathrm{G} 21 \times 5$ & 424.9 & 0.00 & 0.00 & 0.00 & 0.75 & 528.42 & 0.00 & 0.30 & 0.54 & 0.60 \\
\hline $\mathrm{G} 22 \times 5$ & 585.1 & 0.00 & 0.00 & 0.00 & 0.44 & 653.80 & 0.00 & 0.00 & 0.00 & 0.59 \\
\hline $\mathrm{M} 27 \times 5$ & 3062.0 & 0.00 & 0.00 & 0.00 & 0.75 & 3142.02 & 0.00 & 0.00 & 0.00 & 0.69 \\
\hline $\mathrm{G} 29 \times 5$ & 512.1 & 0.00 & 0.00 & 0.00 & 0.74 & 592.10 & 0.00 & 0.00 & 0.00 & 0.53 \\
\hline G32 × 5 & 562.2 & 0.00 & 0.00 & 0.00 & 0.90 & 696.38 & 0.00 & 0.08 & 0.16 & 0.88 \\
\hline $\mathrm{G} 32 \times 5$ & 504.3 & 0.00 & 0.00 & 0.00 & 0.53 & 595.27 & 0.00 & 0.00 & 0.00 & 0.73 \\
\hline $\mathrm{G} 36 \times 5$ & 460.4 & 0.00 & 0.00 & 0.00 & 0.93 & 540.37 & 0.00 & 0.00 & 0.00 & 0.77 \\
\hline $\mathrm{C} 50 \times 5$ & 565.6 & 0.00 & 0.00 & 0.00 & 3.49 & 708.37 & 0.00 & 0.00 & 0.07 & 3.22 \\
\hline $\mathrm{P} 55 \times 5$ & 1112.1 & 0.00 & 0.06 & 0.26 & 6.05 & 1327.06 & 0.00 & 0.05 & 0.93 & 7.31 \\
\hline $\mathrm{C} 75 \times 5$ & 844.4 & 0.00 & 0.02 & 0.07 & 10.62 & 1132.80 & 0.00 & 0.40 & 1.65 & 9.10 \\
\hline $\mathrm{P} 85 \times 5$ & 1622.5 & 0.00 & 0.17 & 1.26 & 12.28 & 1855.55 & 0.00 & 0.12 & 1.82 & 12.20 \\
\hline $\mathrm{D} 88 \times 8$ & 355.8 & 0.00 & 0.00 & 0.00 & 7.33 & 497.60 & 0.08 & 0.18 & 0.32 & 8.69 \\
\hline $\mathrm{C} 100 \times 10$ & 833.4 & 0.00 & 0.32 & 1.58 & 12.92 & 1011.53 & 0.00 & 1.98 & 6.29 & 10.94 \\
\hline $\mathrm{O} 117 \times 14$ & $12,290.3$ & 0.00 & 0.43 & 40.81 & 13.79 & $12,360.70$ & -0.08 & 0.08 & 15.82 & 19.14 \\
\hline M134 × 8 & 5709.0 & 0.00 & 0.16 & 3.37 & 29.29 & 5953.68 & -0.67 & -0.40 & 5.17 & 33.63 \\
\hline $\mathrm{D} 150 \times 10$ & $43,919.9$ & 0.00 & 0.40 & 101.24 & 31.49 & $45,152.90$ & -0.44 & -0.07 & 44.28 & 43.95 \\
\hline Av. & 4585.25 & 0.00 & 0.10 & 9.29 & 8.27 & 4796.78 & -0.07 & 0.17 & 4.82 & 9.56 \\
\hline Md. & 709.25 & 0.00 & 0.00 & 0.00 & 4.77 & 859.95 & 0.00 & 0.03 & 0.43 & 5.27 \\
\hline
\end{tabular}




\subsection{Experiments and Analyses}

In this section, four types of experimental data were created, named LCLIENT, LDEPOT, LVEHICLE, and LCTW and $N \in\{25,50,75,100\}$ in each instance library. The LCLIENT data was divided into four categories, that is, the customers were clustered in three regions with a probability of $60 \%$ and a random distribution type, which were named $\mathrm{CC} 1, \mathrm{CC} 2, \mathrm{CC} 3$, and $\mathrm{CR}$, respectively. The depots were randomly distributed, and the remaining parameters were the same as in Section 5.1. The characteristics of LDEPOT were that the customers were randomly distributed, but the depots had four separate methods, that is, all the depots were distributed in three regions and one random distribution type, which were named DC1, DC2, DC3, and DR respectively. In the LVEHICLE case, the depots and the customers were randomly distributed, but the types of vehicles were different, namely L1, L2, M, and multiple types. The data characteristics of LCTW were the random distribution of customers and depots, but the time window widths were different, that is, $l_{i}-e_{i}=\lambda \cdot S T_{i}$, and $\lambda \in\{1,1.5,2,2.5\}$.

\subsubsection{Impact of Customer Distribution on Cost and Carbon Emissions}

The SESMAM-HH was used to solve LCLIENT and RLRPTWSPDHF problems, which was the model of RLCLRPTWSPDHF without considering carbon emission. The results were used to analyze the impact of customer distribution on logistics cost, carbon emission, and customer satisfaction. Meanwhile, the calculation results of LLEENT and RLRPTWSPDHF were compared to verify the validity of the RLCLRPTWSPDHF. The results are shown in Table 4, where TC is the logistics cost (yuan), FC is the fuel consumption and carbon emission cost (yuan), PF is the sum of the vehicle and customer waiting costs (yuan), $\mathrm{CO}_{2}$ is the carbon emission $(\mathrm{kg}), \mathrm{D}$ is the vehicle travel distance $(\mathrm{km})$, CWT is the customer waiting time (minutes), and MT is the algorithm's average running time (seconds). $\triangle \mathrm{TC}, \triangle \mathrm{CO}_{2}, \triangle \mathrm{D}$, and $\triangle \mathrm{CWT}$ are the increments of TC, CO2, D, and CWT values in RLRPTWSPDHF model and corresponding values in the RLRPTWSPDHF model.

It can be seen from the results that $\mathrm{CC}_{1}$ helps to reduce TC and can reduce TC by $3.39 \%, 8.89 \%$, and $8.73 \%$ on average compared with the other three categories. At $\mathrm{N} \leq 75, \mathrm{CO}_{2}$ and $\mathrm{FC}$ were the lowest in the CC1 case, which were $9.12 \%, 24.98 \%$, and $16.89 \%$ lower than CC2, CC3, and CR, respectively; however, when $\mathrm{N}=100$, the advantage of $\mathrm{CC} 1$ was not obvious.

Table 4. Results of two models on LCLIENT.

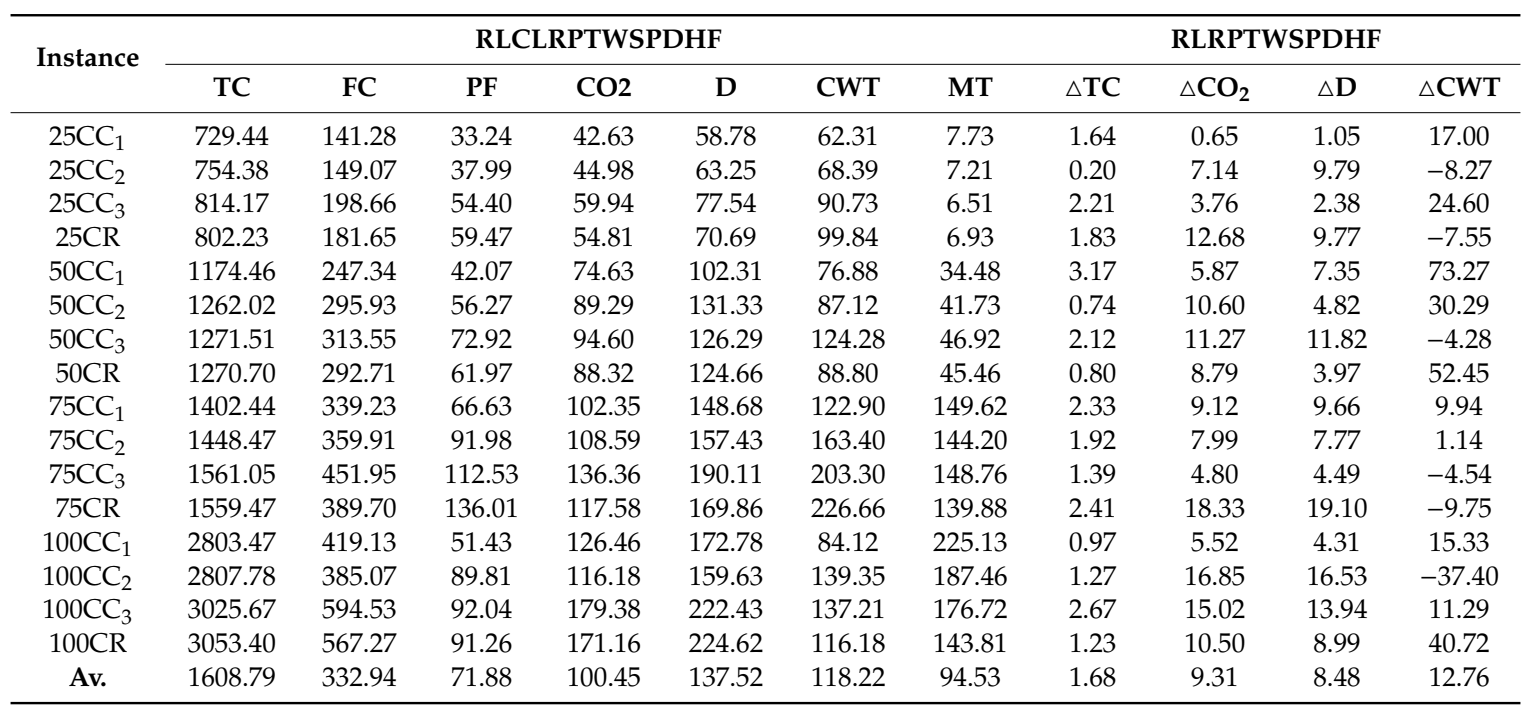

The impact of customer location on PF and CWT values was similar to its impact on TC, which means that $\mathrm{CC} 1$ helped to reduce customer waiting times and the corresponding penalty costs. The impact of customer position on $\mathrm{D}$ was similar to the influence of customer distribution on $\mathrm{CO}_{2}$ and FC, which means CC1 helps to reduce driving distance, but when $\mathrm{N}=100, \mathrm{CC} 2$ is in first place 
with $159.63 \mathrm{~km}$, which is lower than the $172.78 \mathrm{~km}$ of CC1. At the same time, compared with the RLRPTWSPDHF model, the RLCLRPTWSPDHF model can reduce TC by $1.68 \%, \mathrm{CO}_{2}$ by $9.31 \%$, D by $8.48 \%$ and C-WT by $12.76 \%$.

\subsubsection{The Impact of Time Window on Cost and Carbon Emissions}

Table 5 shows the calculation results of the LCTW examples with four different time windows $(\lambda \in\{1,1.5,2,2.5\})$. The results were used to analyze the effects of time windows on $T C, R C, P F, C O_{2}$, $D$, and $C W T$. In Table 5, $\triangle T C, \triangle R C, \triangle P F, \triangle C O_{2}, \triangle D$, and $\triangle C W T$ are the amount of change in $T C, R C$, $P F, C_{2}, D$, and $C W T$ when $\lambda=1$. The bold font is the amount of increase, and the non-bold font is the amount of reduction.

From the results in the table, it can be seen that when $\lambda=1.5$, it can reduce the average TC by $3.04 \%, R C$ by $2.48 \%$, PF by $9.91 \%, C_{2}$ by $1.59 \%, D$ by $4.70 \%$, and $C W T$ by $6.22 \%$; when $\lambda=2$, it can reduce the average TC by $4.86 \%, R C$ by $3.23 \%, P F$ by $31.59 \%, C_{2}$ by $0.33 \%, D$ by $4.77 \%$, and $C W T$ by $30.13 \%$; when $\lambda=2.5$, it can reduce the average $T C$ by $6.59 \%, R C$ by $4.92 \%$, $P F$ by $42.44 \%, \mathrm{CO}_{2}$ by $-1.12 \%$, $D$ by $3.74 \%$, and CWT by $43.35 \%$.

Figure 5 is a graph showing the trend in the average values of the six types of parameters under the four types of $\lambda$ values. Figure 5 shows that $\triangle T C, \triangle R C, \triangle P F$, and $\triangle C W T$ gradually increased with an increase in the $\lambda$ value, but $\mathrm{CO}_{2}$ gradually reduced from $1.59 \%$ to $1.22 \%$. In addition, $\Delta D$ increased by $0.07 \%$ when $\lambda$ increased from 1.5 to 2 , but decreased sharply by $1.3 \%$ when $\lambda$ increased from 2 to 2.5. This indicates that the time window has a great influence on the choice of distribution center and service vehicle.

Table 5. Results of LCTW based on four $\lambda$ values.

\begin{tabular}{|c|c|c|c|c|c|c|c|c|c|c|c|c|}
\hline \multirow{2}{*}{ Instance } & \multicolumn{6}{|c|}{$\lambda=1$} & \multicolumn{6}{|c|}{$\lambda=1.5$} \\
\hline & TC & RC & PF & $\mathrm{CO}_{2}$ & D & CWT & $\triangle \mathrm{TC}$ & $\triangle \mathbf{R C}$ & $\triangle \mathbf{P F}$ & $\triangle \mathrm{CO}_{2}$ & $\triangle \mathbf{D}$ & $\triangle \mathrm{CWT}$ \\
\hline $25-1$ & 1130.4 & 885.9 & 51.0 & 58.4 & 91.4 & 75.2 & 1.55 & 2.80 & 17.04 & 0.74 & 9.74 & 41.52 \\
\hline $25-2$ & 841.8 & 561.1 & 58.7 & 67.0 & 95.6 & 99.7 & 1.89 & 5.52 & 50.68 & 6.58 & 15.13 & 72.95 \\
\hline $25-3$ & 872.7 & 585.9 & 84.2 & 61.1 & 91.8 & 141.3 & 2.03 & 0.00 & 14.17 & 2.85 & 2.76 & 18.65 \\
\hline $50-1$ & 1834.3 & 1366.0 & 142.3 & 98.4 & 131.9 & 245.0 & 1.92 & 2.27 & 12.82 & 4.32 & 1.09 & 9.05 \\
\hline $50-2$ & 1415.5 & 909.8 & 142.1 & 109.7 & 150.1 & 256.5 & 2.97 & 3.41 & 13.80 & 2.37 & 3.15 & 8.31 \\
\hline $50-3$ & 1786.5 & 1359.8 & 106.9 & 96.5 & 137.7 & 181.8 & 2.11 & 0.00 & 23.44 & 3.96 & 5.93 & 28.24 \\
\hline $75-1$ & 2235.1 & 1739.5 & 126.5 & 111.3 & 161.3 & 212.9 & 9.25 & 13.63 & 8.74 & 11.24 & 11.63 & 5.09 \\
\hline $75-2$ & 1760.9 & 1089.5 & 150.7 & 157.1 & 221.5 & 262.8 & 6.40 & 3.41 & 28.42 & 6.28 & 7.71 & 28.92 \\
\hline $75-3$ & 2146.1 & 1521.4 & 206.5 & 126.2 & 171.3 & 350.2 & 2.14 & 1.63 & 17.66 & 3.67 & 1.28 & 13.90 \\
\hline $100-1$ & 2880.3 & 2137.8 & 202.6 & 162.9 & 242.3 & 354.1 & 2.64 & 0.87 & 17.68 & 4.00 & 5.21 & 19.72 \\
\hline $100-2$ & 2738.0 & 2069.7 & 188.5 & 144.8 & 211.6 & 293.3 & 2.32 & 0.00 & 33.65 & 0.00 & 0.00 & 43.24 \\
\hline $100-3$ & 2862.9 & 2144.0 & 170.9 & 165.3 & 231.9 & 239.6 & 1.23 & 3.82 & 16.31 & 16.26 & 18.59 & 13.98 \\
\hline \multirow[t]{2}{*}{ Av. } & 1875.4 & 1364.2 & 135.9 & 113.2 & 161.5 & 226.0 & 3.04 & 2.48 & 9.91 & 1.59 & 4.70 & 6.22 \\
\hline & \multicolumn{6}{|c|}{$\lambda=2$} & \multicolumn{6}{|c|}{$\lambda=2.5$} \\
\hline $25-1$ & 2.90 & 2.80 & 12.90 & 0.74 & 9.74 & 0.95 & 3.68 & 2.80 & 33.25 & 0.09 & 8.95 & 26.62 \\
\hline $25-2$ & 3.58 & 5.52 & 26.38 & 6.58 & 15.13 & 44.37 & 4.99 & 5.52 & 6.13 & 6.58 & 15.13 & 20.55 \\
\hline $25-3$ & 4.49 & 4.23 & 25.20 & 3.37 & 4.34 & 21.53 & 6.30 & 4.23 & 43.99 & 3.37 & 4.34 & 43.93 \\
\hline 50-1 & 3.67 & 2.27 & 48.79 & 10.14 & 3.99 & 48.82 & 5.03 & 2.27 & 51.23 & 3.59 & 0.21 & 53.47 \\
\hline $50-2$ & 5.05 & 3.41 & 40.35 & 4.62 & 0.10 & 37.37 & 6.98 & 3.41 & 30.98 & 6.53 & 11.72 & 27.70 \\
\hline $50-3$ & 3.65 & 2.28 & 36.92 & 1.65 & 5.55 & 34.58 & 4.67 & 1.82 & 50.99 & 1.32 & 6.83 & 55.07 \\
\hline 75-1 & 10.68 & 11.50 & 44.18 & 4.64 & 6.52 & 53.06 & 13.08 & 13.63 & 56.91 & 4.56 & 2.69 & 65.98 \\
\hline $75-2$ & 8.58 & 3.41 & 53.90 & 6.28 & 7.71 & 58.12 & 10.80 & 5.69 & 70.38 & 4.25 & 7.91 & 74.79 \\
\hline $75-3$ & 4.02 & 1.63 & 30.28 & 0.23 & 1.25 & 29.33 & 5.52 & 3.26 & 42.19 & 4.35 & 1.47 & 41.53 \\
\hline $100-1$ & 5.22 & 2.61 & 34.43 & 4.58 & 8.39 & 37.85 & 7.31 & 9.92 & 42.59 & 16.25 & 11.80 & 44.93 \\
\hline $100-2$ & 4.20 & 2.69 & 38.34 & 2.71 & 1.72 & 38.26 & 5.10 & 2.99 & 38.83 & 0.92 & 3.79 & 42.82 \\
\hline $100-3$ & 2.26 & 3.53 & 40.20 & 13.08 & 13.83 & 47.95 & 5.66 & 3.47 & 54.03 & 0.87 & 0.92 & 63.91 \\
\hline Av. & 4.86 & 3.23 & 31.59 & 0.33 & 4.77 & 30.13 & 6.59 & 4.92 & 42.44 & 1.12 & 3.74 & 43.35 \\
\hline
\end{tabular}




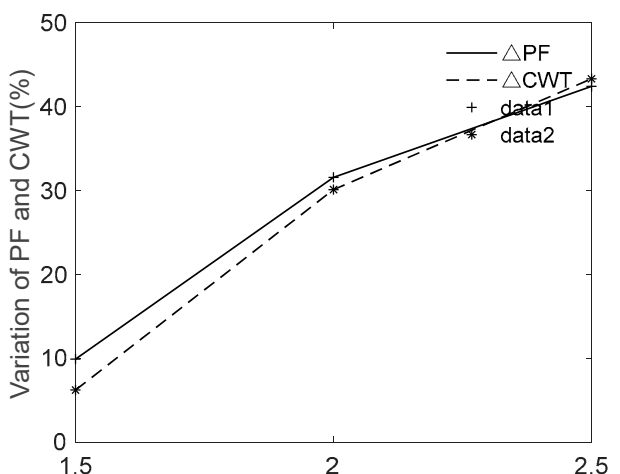

(a) $\lambda$

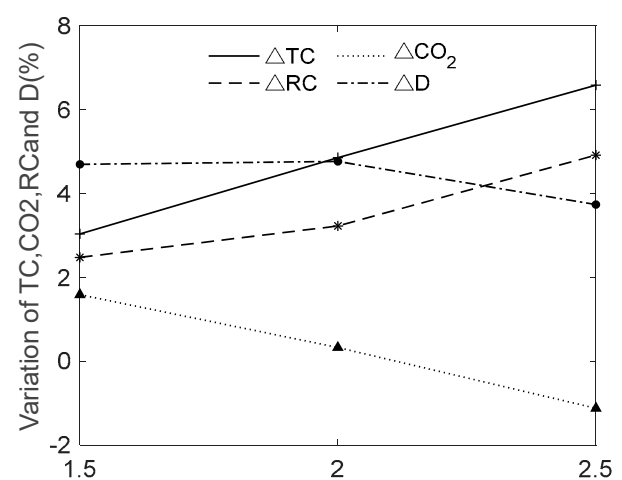

(b) $\lambda$

Figure 5. The trend in the average value of the six types of parameters.

\subsubsection{The Impact of Depot on Costs and Carbon Emissions}

In this section, the proposed algorithm is used to solve the LDEPOT example. Based on the results (see Table 6), the effects of depot distribution and cost on logistics costs, carbon emissions, and customer satisfaction are analyze. Simultaneously, the results are compared with the results of the RRTPTWSPDHF to verify the effectiveness of the proposed model. From the calculation results, the location and cost of the depot had no regular effect on the parameters. Taking TC as an example, $D C 3$ can be reduced by $40.61 \%, 25.12 \%$, and $16.51 \%$ compared to the other three categories; for $P F$ parameters, the average value of $D R$ is lowest. However, compared with the RLRPTWSPDHF model, the RLCRPTWSPDHF model can reduce TC by $1.76 \%, \mathrm{CO}_{2}$ by $13.16 \%$, and $D$ by $11.38 \%$. Comprehensive analysis shows that the RLCRPTWSPDHF model is effective in reducing $\mathrm{TC}, \mathrm{CO}_{2}$, and $D$.

Table 6. Results of two models on LDEPOT.

\begin{tabular}{|c|c|c|c|c|c|c|c|c|c|c|c|}
\hline \multirow{2}{*}{ Instance } & \multicolumn{7}{|c|}{ RLCLRPTWSPDHF } & \multicolumn{4}{|c|}{ RLRPTWSPDHF } \\
\hline & TC & FC & PF & $\mathrm{CO}_{2}$ & $\mathbf{D}$ & CWT & MT & $\triangle \mathrm{TC}$ & $\triangle \mathrm{CO}_{2}$ & $\triangle \mathbf{D}$ & $\triangle \mathrm{CWT}$ \\
\hline $25 \mathrm{DC}_{1}$ & 1420.14 & 223.81 & 35.22 & 67.53 & 82.70 & 42.38 & 5.67 & 1.87 & 14.25 & 5.43 & 164.53 \\
\hline $25 \mathrm{DC}_{2}$ & 1124.73 & 226.06 & 37.56 & 68.21 & 84.56 & 47.35 & 5.68 & 2.02 & 14.86 & 8.09 & 154.12 \\
\hline $25 \mathrm{DC}_{3}$ & 817.45 & 222.55 & 33.80 & 67.15 & 84.25 & 42.46 & 5.57 & 2.89 & 15.42 & 5.41 & 141.85 \\
\hline $25 \mathrm{DR}$ & 969.23 & 222.70 & 35.42 & 67.19 & 83.77 & 45.80 & 5.64 & 3.13 & 17.84 & 10.56 & 154.87 \\
\hline $50 \mathrm{DC}_{1}$ & 2159.69 & 311.72 & 93.92 & 94.05 & 111.53 & 164.16 & 60.36 & 1.13 & 17.99 & 15.73 & -26.53 \\
\hline $50 \mathrm{DC}_{2}$ & 1696.93 & 295.64 & 66.25 & 89.20 & 110.68 & 108.15 & 56.88 & 0.15 & 5.29 & 1.94 & 46.43 \\
\hline $50 \mathrm{DC}_{3}$ & 1242.23 & 286.28 & 101.89 & 86.38 & 105.33 & 185.66 & 59.28 & 1.49 & 12.16 & 9.69 & -17.68 \\
\hline 50DR & 1845.71 & 298.77 & 92.88 & 90.14 & 109.78 & 170.66 & 58.69 & 0.25 & 4.87 & 3.67 & -6.78 \\
\hline $75 \mathrm{DC}_{1}$ & 2490.21 & 440.77 & 128.08 & 132.99 & 189.06 & 220.35 & 162.65 & 1.53 & 14.30 & 16.75 & -43.77 \\
\hline $75 \mathrm{DC}_{2}$ & 1999.16 & 403.59 & 124.20 & 121.77 & 173.31 & 211.99 & 170.16 & 2.13 & 13.32 & 15.86 & -42.60 \\
\hline $75 \mathrm{DC}_{3}$ & 1547.71 & 398.59 & 109.16 & 120.26 & 169.58 & 188.57 & 159.81 & 1.83 & 11.17 & 13.78 & -37.20 \\
\hline $75 \mathrm{DR}$ & 1734.25 & 391.37 & 90.54 & 118.08 & 168.35 & 138.09 & 153.35 & 2.62 & 16.28 & 14.56 & -37.03 \\
\hline $100 \mathrm{DC}_{1}$ & 3734.70 & 521.42 & 124.61 & 157.32 & 222.91 & 207.26 & 221.97 & 1.33 & 11.77 & 10.27 & -5.89 \\
\hline $100 \mathrm{DC}_{2}$ & 2955.58 & 496.55 & 107.97 & 149.82 & 210.60 & 167.98 & 202.27 & 2.00 & 14.83 & 17.59 & -44.85 \\
\hline $100 \mathrm{DC}_{3}$ & 2252.70 & 527.52 & 130.31 & 159.16 & 226.34 & 222.52 & 208.91 & 1.91 & 12.30 & 12.66 & -13.65 \\
\hline 100DR & 2420.71 & 517.14 & 114.90 & 156.03 & 220.66 & 189.57 & 228.46 & 1.97 & 13.89 & 12.19 & -14.15 \\
\hline Av. & 1900.70 & 361.53 & 89.17 & 109.08 & 147.09 & 147.06 & 110.33 & 1.76 & 13.16 & 10.89 & 23.23 \\
\hline Md. & 1789.98 & 351.54 & 97.90 & 106.07 & 139.94 & 169.32 & 106.85 & 1.89 & 14.07 & 11.38 & -13.90 \\
\hline
\end{tabular}

\subsubsection{The Impact of the Fleets on Costs and Carbon Emissions}

This section analyzes the impact of fleets on logistics costs and carbon emissions by solving the LVEHICLE case. The results are shown in Table 7.

As seen from Table 7, HF can obtain the lowest $T C$ and $\mathrm{CO}_{2} . R C$ of $H F$ is smaller than $L 1$ and $M$, but greater than $L 2$, at approximately $1.3 \%$, mainly reflecting the influence of different fleets and quantities on $R C$. Different fleets have no effect on depots selection. PF and CWT values of $L 1$ are the 
smallest, indicating that $L 1$ vehicle plays a key role in customer satisfaction. The reason is that time window parameters may be consistent with $L 1$ vehicle parameters. According to $D$ value analysis, $L 2$ and $M$ have smaller $D$ values, because the vehicle capacity is relatively large and can serve more customers. Figure 6 is a box plot of vehicle capacity utilization. The average full load of HF is $85.92 \%$, which is higher than that of $25.28 \%$ and $105.26 \%$ for L2 and M, but lower than $4.61 \%$ for L1.

As can be seen from the above, using HF can reduce logistics costs and carbon emissions compared with a single type vehicle, but there is no guarantee of obtaining optimal values of waiting time, service distance, and vehicle full load rate. However, it can still be strongly inferred that the HF has huge advantages over a single car model and can better meet the requirements of practical distribution applications.

Table 7. Results of instances with different fleet.

\begin{tabular}{|c|c|c|c|c|c|c|c|c|c|c|c|c|}
\hline \multirow{2}{*}{ Instance } & \multicolumn{6}{|c|}{ HF } & \multicolumn{6}{|c|}{$\mathrm{L}_{1}$} \\
\hline & $\mathrm{TC}$ & RC & PF & $\mathrm{CO}_{2}$ & D & CWT & $\triangle \mathrm{TC}$ & $\triangle \mathrm{RC}$ & $\triangle \mathbf{P F}$ & $\triangle \mathrm{CO}_{2}$ & $\triangle \mathbf{D}$ & $\triangle \mathrm{CWT}$ \\
\hline $25-1$ & 843.0 & 561.1 & 82.7 & 60.1 & 81.0 & 150.3 & 1.54 & 4.42 & -39.90 & 10.66 & 21.81 & -48.95 \\
\hline $25-2$ & 990.3 & 742.1 & 52.0 & 59.2 & 88.1 & 70.7 & 0.85 & -0.84 & -5.59 & 8.95 & 11.73 & 2.57 \\
\hline $25-3$ & 838.9 & 561.1 & 65.3 & 64.1 & 85.5 & 120.3 & 1.21 & 4.42 & -18.88 & -1.10 & 8.61 & -29.45 \\
\hline $50-1$ & 1445.1 & 1059.8 & 66.6 & 96.1 & 123.6 & 104.7 & 3.44 & 5.85 & -53.14 & 7.26 & 14.54 & -91.94 \\
\hline $50-2$ & 1582.1 & 1209.8 & 79.9 & 88.2 & 120.7 & 132.4 & 1.56 & 2.05 & -14.12 & 3.83 & 9.19 & -28.99 \\
\hline $50-3$ & 1552.7 & 1178.8 & 95.1 & 84.1 & 114.7 & 156.9 & 2.19 & 4.73 & -47.24 & 8.28 & 16.89 & -69.46 \\
\hline $75-1$ & 1850.0 & 1383.8 & 74.8 & 118.1 & 171.6 & 102.9 & 2.87 & 3.58 & 11.25 & -1.23 & 5.15 & -18.78 \\
\hline $75-2$ & 1928.7 & 1396.2 & 88.2 & 134.1 & 183.4 & 118.2 & 1.52 & 2.66 & -28.78 & 3.94 & 5.68 & -47.61 \\
\hline $75-3$ & 1524.9 & 1027.6 & 106.8 & 117.8 & 158.1 & 166.2 & 3.40 & 5.43 & -23.64 & 5.47 & 11.02 & -40.65 \\
\hline $100-1$ & 2725.2 & 2094.9 & 123.7 & 152.9 & 201.4 & 166.2 & 5.88 & 7.10 & -2.42 & 2.87 & 12.76 & -55.55 \\
\hline $100-2$ & 2911.4 & 2301.5 & 127.9 & 145.4 & 204.4 & 220.3 & 2.49 & 5.12 & -34.62 & -0.19 & 8.13 & -67.03 \\
\hline $100-3$ & 3256.9 & 2657.7 & 125.0 & 143.1 & 187.7 & 209.7 & 5.78 & 6.99 & -2.65 & 1.20 & 17.91 & -56.58 \\
\hline \multirow[t]{2}{*}{ Av. } & 1787.4 & 1347.9 & 90.7 & 105.3 & 143.4 & 143.2 & 2.73 & 4.29 & -21.64 & 4.16 & 11.95 & -46.03 \\
\hline & \multicolumn{6}{|c|}{$\mathbf{L}_{2}$} & \multicolumn{6}{|c|}{$\mathbf{M}$} \\
\hline $25-1$ & 1.03 & 2.21 & -2.38 & -0.87 & -2.63 & -2.26 & 9.80 & 9.15 & -3.97 & 17.35 & -2.63 & -4.38 \\
\hline $25-2$ & 1.62 & -2.51 & 42.63 & 6.35 & -4.06 & 83.10 & 9.39 & 2.74 & 42.63 & 25.72 & -4.06 & 83.10 \\
\hline $25-3$ & 2.29 & 2.21 & 0.00 & 3.22 & 0.00 & 0.00 & 11.85 & 9.15 & 0.00 & 22.62 & 0.00 & 0.00 \\
\hline $50-1$ & 1.00 & -4.68 & 96.56 & -0.07 & -10.33 & 139.62 & 8.73 & 0.84 & 131.77 & 9.27 & -16.66 & 180.24 \\
\hline $50-2$ & 2.30 & -0.51 & 25.04 & 7.70 & 0.24 & 41.06 & 10.11 & 5.12 & 29.02 & 25.57 & -1.13 & 45.36 \\
\hline $50-3$ & 0.96 & -1.58 & 14.45 & 7.07 & -0.03 & 29.34 & 8.14 & 3.38 & 13.97 & 26.29 & -0.48 & 29.62 \\
\hline $75-1$ & 1.53 & 0.00 & 32.05 & 1.12 & -4.23 & 56.70 & 9.48 & 3.20 & 90.17 & 16.26 & -8.86 & 149.68 \\
\hline $75-2$ & 2.02 & -0.89 & 10.74 & 9.42 & -1.90 & 41.51 & 10.25 & 2.28 & 84.95 & 20.47 & -9.80 & 154.29 \\
\hline $75-3$ & 2.32 & -3.62 & 75.01 & -1.93 & -9.74 & 105.75 & 12.43 & 4.91 & 79.80 & 13.78 & -11.87 & 108.46 \\
\hline $100-1$ & 0.38 & -3.55 & 61.33 & 1.76 & -6.05 & 121.03 & 8.32 & 2.03 & 49.55 & 24.25 & -1.87 & 102.06 \\
\hline $100-2$ & 0.58 & -1.35 & 37.77 & -0.11 & -6.10 & 49.29 & 8.60 & 3.73 & 57.05 & 19.02 & -5.09 & 71.71 \\
\hline $100-3$ & 0.43 & -1.40 & 24.67 & 4.31 & 2.19 & 35.75 & 5.99 & 1.00 & 69.93 & 17.11 & -5.73 & 90.30 \\
\hline Av. & 1.37 & -1.30 & 34.82 & 3.16 & -3.55 & 58.41 & 9.42 & 3.96 & 53.74 & 19.81 & -5.68 & 84.20 \\
\hline
\end{tabular}

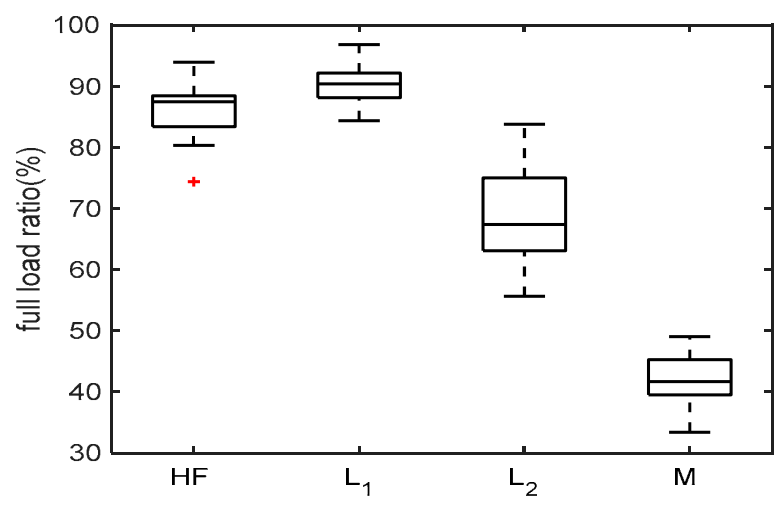

Figure 6. The full load ratios of different fleets. 


\section{Conclusions}

This paper comprehensively studied the impact of HF, simultaneous delivery, customer time windows, and speed regionalization on logistics costs, carbon emissions, and customer satisfaction. It also proposed the RLCLRPTWSPDHF model.

At the same time, a new hyper-heuristic solving algorithm, named SESMAM-AA, was proposed. This algorithm uses the second exponential smoothing method to predict the recent performance of low-level operators. The operator is adaptively selected according to the performance of the algorithm in the near term (quality operator/inferior operator). Finally, the FRR-MAB method is used to evaluate the operator's credit value, and the roulette probability distribution is used to select the appropriate and accurate low-level operator.

This paper conducted three types of experiments. The first was to verify the correctness of the model and the accuracy of the algorithm. The second experiment was to verify the efficiency of the algorithm by solving the CLRP/CLRPSPD benchmarks. The purpose of the third experiment was to analyze the influence of customer parameters, depot parameters, and fleet types on logistics costs, carbon emissions, and customer satisfaction. At the same time, the validity of the model was verified. The experimental results show that the RLCLRPTWSPDHF model can effectively reduce distribution costs, reduce carbon emissions, and protect the environment.

According to the analysis of experimental results, the following conclusions are drawn:

1. The proposed algorithm has high accuracy in solving small-scale examples;

2. The SESMAM-AA algorithm can obtain high quality solutions in reasonable computing time with high efficiency and robustness;

3. The HF can reduce logistics costs, carbon emissions, and service distances, and can better meet actual distribution requirements;

4. The distribution location of customers and depots has a great influence on each function value;

5. The RLCLRPTWSPDHF model can reduce TC, $\mathrm{CO}_{2}$, and $\mathrm{D}$ better than the traditional model, but there is no guarantee that CWT will always be reduced to improve customer satisfaction.

In summary, the research in this paper has important practical and theoretical significance for logistics enterprises seeking to reduce logistics costs and protect the ecological environment, and has an important supporting role in sustainable development and energy conservation and emission reduction policies. However, in addition to considering operating costs, customer satisfaction is also a major concern of logistics companies. Therefore, the main task in the next step will be to make customer satisfaction the second optimization goal to build a multi-objective model and, at the same time, to design a multi-objective hyper-heuristic algorithm to obtain the Pareto set. Finally, this paper analyzed customer satisfaction under the premise of soft time windows. To improve customer satisfaction, hard time windows should be researched.

Author Contributions: Conceptualization, Y.Z. and C.Z.; methodology, C.Z.; software, C.Z.; validation, L.L., Y.Z. and C.Z.; formal analysis, Y.Z.; investigation, L.L.; resources, L.L.; data curation, L.L.; writing-original draft preparation, Y.Z.; writing-review and editing, C.Z.; visualization, C.Z.; supervision, C.Z.; project administration, Y.Z.; funding acquisition, Y.Z. and C.Z.

Funding: This research was funded by the National Natural Science Foundation of China grant numbers 61572438, Science Technology plan project of Zhejiang grant number 2017C33224. This support is gratefully acknowledged. Thanks for the reviewers and editors who provided valuable comments and suggestions for revising this paper.

Conflicts of Interest: The authors declare no conflict of interest.

\section{References}

1. Shi, X.H.; Zhang, Z.R.; Wang, B. Particle swarm optimization and its application in location routing problem. Comput. Sci. 2012, 39, 270-272. [CrossRef]

2. Zhang, C.M.; Zhao, Y.W.; Zhang, J.L.; Leng, L.L. Location and routing problem with minimizing carbon. Comp. Integr. Manuf. Syst. 2017, 23, 2768-2777. [CrossRef] 
3. Shi, Z.; Fu, Z. Distribution location-routing problem of heterotypic vehicles and its algorithms. Comput. Sci. 2015, 42, 245-250. [CrossRef]

4. Huang, L.X.; Zhao, J. Optimization of location-routing problem in regional hazardous waste management systems with path-bath formulation. Comput. Integr. Manuf. Syst. 2016, 22, 2023-2034. [CrossRef]

5. Li, C.B.; Zhang, F.M. Reverse logistics network optimization of integrated location-routing-inventory problem. Comput. Integr. Manuf. Syst. 2014, 20, 1793-1798. [CrossRef]

6. Pan, S.; Ballot, E.; Fontane, F. The reduction of greenhouse gas emissions from freight transport by pooling supply chains. Int. J. Prod. Econ. 2013, 143, 86-94. [CrossRef]

7. Urquhart, N.; Scott, C.; Hart, E. Using an evolutionary algorithm to discover low $\mathrm{CO}_{2}$ tours within a travelling salesman problem. Appl. Evolut. Comput. 2010, 6025, 421-430. [CrossRef]

8. Demir, E.; Bektas, T.; Laporte, G. A comparative analysis of several vehicle emission models for road freight transportation. Transp. Res. Part D 2011, 16, 347-357. [CrossRef]

9. Jovicic, N.M.; Boskovic, G.B.; Vujic, G.V.; Jovičić, G.R.; Despotović, M.Z.; Milovanović, D.M.; GORDIĆ, D.R. Route optimization to increase energy efficiency and reduce fuel consumption of communal vehicles. Therm. Sci. 2010, 14, 67-78. [CrossRef]

10. Ecological Transport Information Tool for Worldwide Transports, 2011. Available online: https://www. ecotransit.org/download/EcoTransIT_World_Methodology_Data_100521.pdf (accessed on 5 May 2019).

11. Bowyer, D.P.; Biggs, C.; Akçelik, R. Guide to fuel consumption analysis for urban traffic management. Aust. Road Res. Board Transp. Res. 1985, 56, 31-34.

12. NAEI. Emission Factors. United Kingdom: National Atmospheric Emissions Inventory, 2012. Available online: http://naei.beis.gov.uk/ (accessed on 12 April 2019).

13. Bandeira, J.; Almeida, T.G.; Khattak, A.J.; Rouphail, N.M.; Coelho, M.C. Generating emissions information for route selection: Experimental monitoring and routes characterization. J. Intell. Transp. Syst. 2013, 17, 3-17. [CrossRef]

14. Wygonik, E.; Goodchild, A. Using a GIS-based emissions minimization vehicle routing problem with time windows (EVRPTW) model to evaluate $\mathrm{CO}_{2}$ emissions and cost trade-offs in a case study of an urban delivery system. In Proceedings of the Transportation Research Board 90th Annual Meeting, Washington, DC, USA, 23-27 January 2011.

15. A Comparative Empirical Analysis of Eco-Friendly Routes During Peak and Off-Peak Hours. Available online: https://www.researchgate.net/profile/Jorge_Bandeira/publication/265040840_A_ COMPARATIVE_EMPIRICAL_ANALYSIS_OF_ECO-FRIENDLY_ROUTES_2_DURING_PEAK_ AND_OFF-PEAK_HOURS_3_4_5_24_25_33_34/links/546e20620cf2b5fc17604450/A-COMPARATIVEEMPIRICAL-ANALYSIS-OF-ECO-FRIENDLY-ROUTES-2-DURING-PEAK-AND-OFF-PEAK-HOURS-34-5-24-25-33-34.pdf (accessed on 10 May 2019).

16. Kang, J.; Ma, T.; Ma, F.; Huang, J. Link-based emission model for eco routing. In Proceedings of the International Conference on Its Telecommunications, St. Petersburg, Russia, 23-25 August 2011.

17. Koç, Ç.; Bektaş, T.; Jabali, O.; Laporte, G. The impact of depot location, fleet composition and routing on emissions in city logistics. Transp. Res. Part B 2016, 84, 81-102. [CrossRef]

18. Hoff, A.; Andersson, H.; Christiansen, M.; Hasle, G.; Løkketangen, A. Industrial aspects and literature survey: fleet composition and routing. Comput. Oper. Res. 2010, 37, 2041-2061. [CrossRef]

19. Kopfer, H.W.; Kopfer, H. Emissions Minimization Vehicle Routing Problem in Dependence of Different Vehicle Classes. In Dynamics in Logistics; Springer: Berlin, Germany, 2013; pp. 49-58.

20. Kopfer, H.W.; Schonberger, J.; Kopfer, H. Reducing greenhouse gas emissions of a heterogeneous vehicle fleet. Flex. Serv. Manuf. J. 2014, 26, 221-248. [CrossRef]

21. Koç, Ç.; Bektaş, T.; Jabali, O.; Laporte, G. The fleet size and mix pollution-routing problem. Transp. Res. Part B 2014, 70, 239-254. [CrossRef]

22. $\mathrm{Li}, \mathrm{J} . ; \mathrm{Fu}, \mathrm{P} . \mathrm{H}$. Heterogeneous fixed fleet low-carbon routing problem and algorithm. Comput. Integr. Manuf. Syst. 2013, 19, 1352-1362.

23. Pitera, K.; Sandoval, F.; Goodchild, A. Evaluation of emissions reduction in urban pickup systems heterogeneous fleet case study. Transp. Res. Rec. 2011, 2224, 8-16. [CrossRef]

24. Xiao, Y.Y.; Konak, A. The heterogeneous green vehicle routing and scheduling problem with time-varying traffic congestion. Transp. Res. Part E 2016, 88, 146-166. [CrossRef] 
25. Bigazzi, A.Y.; Figliozzi, M.A. Congestion and emissions mitigation: A comparison of capacity, demand, and vehicle based strategies. Transp. Res. Part D 2012, 17, 538-547. [CrossRef]

26. Barth, M.; Boriboonsosin, K. Real-world $\mathrm{CO}_{2}$ impacts of traffic congestion. Transp. Res. Rec. 2008, 2058, 163-171. [CrossRef]

27. Jabali, O.; Woensel, T.V.; Kok, A.G.D. Analysis of travel times and $\mathrm{CO}_{2}$ emissions in time dependent vehicle routing. Prod. Oper. Manag. 2012, 21, 1060-1074. [CrossRef]

28. Kuo, Y.Y. Using simulated annealing to minimize fuel consumption for the time-dependent vehicle routing problem. Comput. Ind. Eng. 2010, 59, 157-165. [CrossRef]

29. Figliozzi, M.A. The time dependent vehicle routing problem with time windows: Benchmark problems, an efficient solution algorithm, and solution characteristics. Transp. Res. Part E 2012, 48, 616-636. [CrossRef]

30. Soysal, M.; Bioemhof-Ruwaard, J.M.; Bektaş, T. The time-dependent two-echelon capacitated vehicle routing problem with environmental considerations. Int. J. Prod. Econ. 2015, 164, 366-378. [CrossRef]

31. Franceschetti, A.; Honhon, D.; van Woensel, T.; Bektaş, T.; Laporte, G. The time-dependent pollution-routing problem. Transp. Res. Part B 2013, 56, 265-293. [CrossRef]

32. Kazemian, I.; Aref, S. A green perspective on capacitated time-dependent vehicle routing problem with time windows. Mathematics 2015, 2, 20-38. [CrossRef]

33. Mirnohammadi, S.H.; Tirkolaee, E.B.; Goli, A.; Dehnavi-Arani, S. The periodic green vehicle routing problem with considering if time-dependent urban traffic and time windows. Int. J. Optim. Civ. Eng. 2017, 7, 143-156.

34. Poonthalir, G.; Nadarajan, R. A fuel efficient green vehicle routing problem with varying speed constraint. Expert Syst. Appl. 2018, 100, 131-144. [CrossRef]

35. Gendreau, M.; Ghiani, G.; Guerriero, E. Time-dependent routing problems: A review. Comput. Oper. Res. 2015, 64, 189-197. [CrossRef]

36. Shaw, P. Using constraint programming and local search methods to solve vehicle routing problems. In Proceedings of the Principles and Practice of Constraint Programming, Pisa, Italy, 26-30 October 1998; pp. 417-431.

37. DFT. Setting Local Speed Limits. United Kingdom: Department for Transport, 2013. Available online: https://assets.publishing.service.gov.uk/government/uploads/system/uploads/attachment_data/file/ 63975/circular-01-2013.pdf (accessed on 15 March 2019).

38. Cowling, P.; Kendall, G.; Soubeiga, E. A hyper-heuristic approach to scheduling a sales summit. In Proceedings of the International Conference on the Practice and Theory of Automated Timetabling, Konstanz, Germany, 16-18 August 2000; pp. 176-190. [CrossRef]

39. Burke, E.K.; Gendreau, M.; Hyde, M.; Kendall, G.; Özcan, E.; Qu, R. Hyper-heuristics: A survey of the state of the art. J. Oper. Res. Soc. 2013, 64, 1695-1724. [CrossRef]

40. Li, K.; Fialho, A.; Kwong, S.; Zhang, Q. Adaptive operator selection with bandits for a multiobjective evolutionary algorithm based on decomposition. IEEE Trans. Evolut. Comput. 2014, 18, 114-130. [CrossRef]

41. Zhou, Y.Q.; Luo, Q.F.; Chen, H.; He, A.P.; Wu, J.Z. A discrete invasive weed optimization algorithm for salve traveling salesman problem. Neurocomputing 2015, 151, 1227-1236. [CrossRef]

42. Barreto, S.; Ferreira, C.; Paixão, J.; Santos, B.S. Using clustering analysis in a capacitated location-routing problem. Eur. J. Oper. Res. 2007, 179, 968-977. [CrossRef]

43. Yu, V.F.; Lin, S.Y. Solving the location-routing problem with simultaneous pickup and delivery by simulating annealing. Int. J. Prod. Res. 2016, 54, 526-549. [CrossRef]

44. Lopes, R.B.; Ferreria, C.; Santos, B.S. A simple and effective evolutionary algorithm for the capacitated location-routing problem. Comput. Oper. Res. 2016, 70, 155-162. [CrossRef]

(C) 2019 by the authors. Licensee MDPI, Basel, Switzerland. This article is an open access article distributed under the terms and conditions of the Creative Commons Attribution (CC BY) license (http://creativecommons.org/licenses/by/4.0/). 